\title{
Leukoencephalopathy and Akinetic Mutism in a Married Couple: A Probable Association with Oriental Medicine
}

\author{
Ick-Sung Lee, Young-Do Kim, Se-Yoon Kwon, Joong-Seok Kim \\ Department of Neurology, College of Medicine, The Catholic University of Korea, Seoul, Korea
}

\author{
Received February 10, 2010 \\ Revised July 6, 2010 \\ Accepted July 6, 2010

\section{Correspondence} \\ Joong-Seok Kim, MD, PhD \\ Department of Neurology, \\ College of Medicine, \\ The Catholic University of Korea, \\ Seoul St. Mary's Hospital, \\ 505 Banpo-daero, Seocho-gu, \\ Seoul 137-701, Korea \\ Tel $+82-2-2258-6078$ \\ Fax +82-2-599-9686 \\ E-mail neuronet@catholic.ac.kr
}

Background Oriental medicines have been associated with severe psychiatric, neurological, and other adverse medical events. These medicines occasionally cause a typical reversible toxic encephalopathy, but most such cases are not recognized because these adverse events are complex and are associated with other systemic signs and symptoms.

Case Report We describe a married couple with rapid progressive cognitive impairment and akinetic mutism after taking the same oriental medicines on the same day. Brain magnetic resonance images of the couple showed typical leukoencephalopathy in the periventricular white matter and basal ganglia regions, bilaterally.

Conclusions The development of neurobehavioral symptoms and toxic leukoencephalopathy in both patients following the ingestion of oriental medicines is suggestive of a cause-and-effect association, although such a relationship needs to be verified.

J Clin Neurol 2011;7:223-226

\section{Introduction}

The term "toxic leukoencephalopathy" encompasses various diseases that may injure and cause structural alteration of the brain white matter; the insults may be toxic or metabolic secondary to chemotherapy or immunosuppressive therapy, environmental, or infectious in origin. ${ }^{1,2}$ White-matter tracts devoted to higher cerebral function may be involved, causing clinical features that range from inattention, forgetfulness, and changes in personality, to dementia, coma, and death. ${ }^{1}$

Oriental medicines have been associated with severe psychiatric, neurological, and other adverse medical events. It is therefore important that psychiatrists, neurologists, and associated health-care providers are aware of such side effects. Occasionally, these medicines can cause a typical, reversible toxic encephalopathy; however, most such cases are not recognized because these adverse events are complex and are associated with other systemic signs and symptoms. ${ }^{3}$

(a) This is an Open Access article distributed under the terms of the Creative Commons Attribution Non-Commercial License (http://creativecommons.org/licenses/by-nc/3.0) which permits unrestricted non-commercial use, distribution, and reproduction in any medium, provided the original work is properly cited.
We describe herein a married couple with progressive dementia, akinetic mutism, and toxic leukoencephalopathy, as demonstrated on brain magnetic resonance imaging (MRI), after a single dose of the same herbal medicine.

\section{Case Report}

\section{The wife}

A 61-year-old woman with a 3-year history of total thyroidectomy due to thyroid papillary cancer was admitted to hospital with behavioral changes over the previous 5 days. Fifteen days prior to her admission, the patient had taken one pack of herbs as a tonic medicine, which contained Astragalus, $A n$ gelica, Cnidium, Chrysanthemum, Aucklandia, Atractylodes, Bupleurum, and Cimicifuga species, ginger, cornus fruit, citrus peel, licorice, and other unknown adjuvants. The next morning the patient developed severe nausea, vomiting, and indigestion; these symptoms persisted for 3 days. Over a period of several days thereafter, the patient had poor oral intake, generalized aches and weakness. Five days before admission the patient developed inappropriate and incongruent speech, poor comprehension, disorientation, and repetitive meaningless behavior. One day before admission she developed aki- 
netic mutism, lethargy, and withdrawal. There was no personal or family history of neurological diseases. The patient had worked as a teacher for an alternative school in a rural environment and there was no history of exposure to carbon monoxide.

On neurological examination the patient was alert, but she did not respond to verbal or sensory stimuli. There was no spontaneous movement during the examination. The patient's motor responses to pain were contraction and symmetric muscle spasticity. The deep-tendon reflexes were hyperactive in all extremities. There were no abnormal movements, neck stiffness, or pathological reflexes.

Laboratory studies including a complete blood count, blood chemistry, thyroid function testing, venereal disease researchlaboratory testing, autoimmune vasculitis markers, HIV antibodies, vitamin $\mathrm{B} 12$, and folate all produced normal results. The cerebrospinal fluid had a normal opening pressure with a leukocyte count of $1 / \mathrm{mm}^{3}$, a protein level of $45.1 \mathrm{mg} / \mathrm{dL}$, and a glucose level of $62 \mathrm{mg} / \mathrm{dL}$. Cerebrospinal fluid smears and cultures were negative for bacteria, virus, mycobacterium, and fungus, as was the Western blot test for the 14-3-3 protein. The results of the following laboratory tests were also either normal or negative: thyroid stimulation hormone receptor antibody level, antimicrosomal antibody level, pituitary hormone level, ammonia and toxicology screening (paraquat, amphetamine, cocaine, tetrabromophenolphthalein ethyl ester, and ethanol). Hair and serum assays for the following heavy metals were either normal or negative: cadmium, cobalt, molybdenum, uranium, beryllium, sulfate, chromium, aluminum, copper, lead, mercury, selenium, arsenic, nickel, and silver. A direct assay for herbal medicine using the Cyantesmo kit did not reveal cyanide. ${ }^{4}$

An electroencephalographic examination revealed generalized delta activity at 3-6 Hz bilaterally, but no epileptiform discharge was observed. Brain MRI revealed diffuse, symmetric, high signal intensities in both the periventricular white matter and basal ganglia medial portion in T2-weighted and fluid-attenuated inversion recovery (FLAIR) images, while diffusion-weighted, T1-weighted, and postcontrast T1-weighted images were normal (Fig. 1A). The follow-up MRI performed 1 month after admission revealed more extensive symmetrical high signal intensities in T2-weighted and FLAIR images (Fig. 1B). On the diffusion-weighted images, the lesion had a high signal intensity, with a low signal intensity on the apparent diffusion coefficient map (Fig. 1C).

The patient's mental state deteriorated rapidly and she became stuporous 1 week after admission; 1 month later her condition gradually improved and she was alert without awareness. Six months after discharge the patient was able to ambulate in a wheelchair and communicate with family members.

\section{The husband}

A 68-year-old man (the husband of case 1) was found wandering in the hospital construction site 8 days after his wife's admission. Two days previously he had become lost after patient visiting hours. He had taken the same oriental medicine as his wife, on the same day. The next morning he developed nausea and vomiting to a lesser extent. Over a period of days he developed generalized aches and weakness. There was no history of medical or neurological diseases. The patient had worked as a minister in a rural environment.

On examination the patient was alert but without awareness. Almost no meaningful speech was elicited. His motor responses to pain were contraction and symmetric muscle spasticity. His deep-tendon reflexes were hyperactive in all extremities; there were no abnormal movements, neck stiffness, or pathological reflexes.

The same laboratory studies were performed as for case 1, and they revealed no abnormalities. The brain MRI revealed hyperintense lesions of the white matter bilaterally on T2weighted, FLAIR, and diffusion-weighted images, similar to case 1 (Fig. 2A). Follow-up MRI performed 1 month after admission revealed more extensive hyperintense lesions (Fig. 2B).

The progression of this patient's neurological condition was similar to that of case 1 , improving gradually after 1 month. He was able to ambulate with assistance from his family after 6 months.

\section{Discussion}

Toxic leukoencephalopathy is a disorder that is related primarily to the use of leukotoxic therapeutic agents, illicit drug use, and occupational exposure to toxins. Most patients typically present with neurobehavioral dysfunction reflecting diffuse involvement of the cerebral white matter. ${ }^{1} \mathrm{~A}$ heterogeneous array of causes has been described that can lead to toxic leukoencephalopathy with a similar clinical presentation. ${ }^{5}$

There have been few case reports of toxic encephalopathy associated with the use of oriental medicine. ${ }^{6,7}$ However, in most cases of toxic encephalopathy associated with oriental medicine, the direct toxicity of each herb and the toxic effects of a mixture of herbs have been neither verified nor reproduced. Contamination by heavy metals or toxins during the manufacturing process may also play a role.

The findings of neurobehavioral symptoms and unusual white-matter signal changes in the two cases reported herein following the ingestion of oriental medicines suggest a possible relationship, although no heavy metals or toxins were detected. It is of course possible that the toxic leukoencephalopathy is unrelated to ingestion of the oriental medicine. In addition, some unknown event or environmental exposure cannot be 
Lee IS et al.
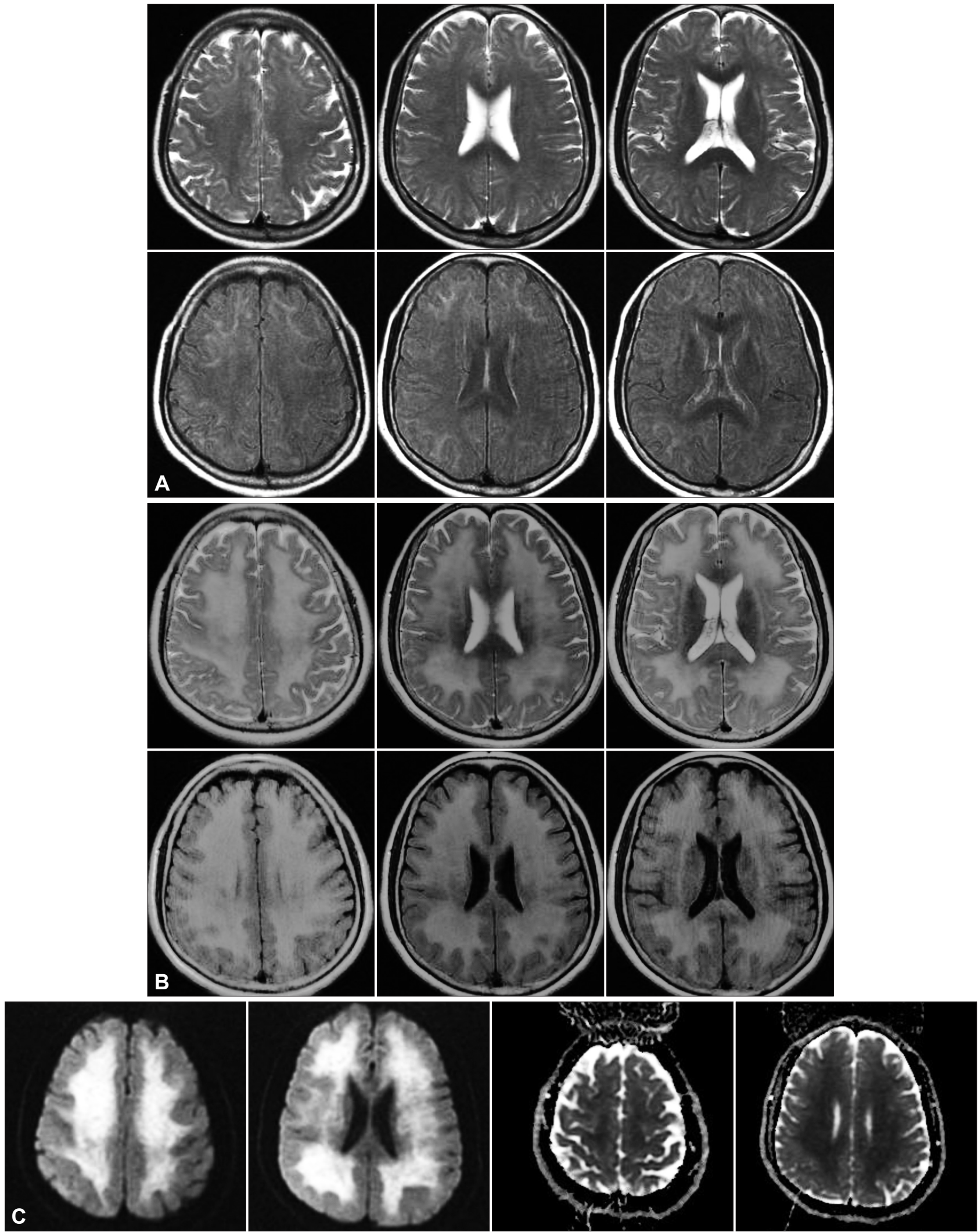

Fig. 1. Case 1; the wife. A: T2-weighted and fluid attenuated inversion recovery (FLAIR) magnetic resonance imaging (MRI) showing hyperintense lesions in the periventricular and deep white matter, and basal ganglia, bilaterally. B: Follow-up MRI performed 1 month after admission revealed more extensive symmetrical high signal intensities in T2-weighted and FLAIR images. C: The lesion had a high signal intensity on diffusion-weighted images, with a low signal intensity on the apparent-diffusion-coefficient map. 

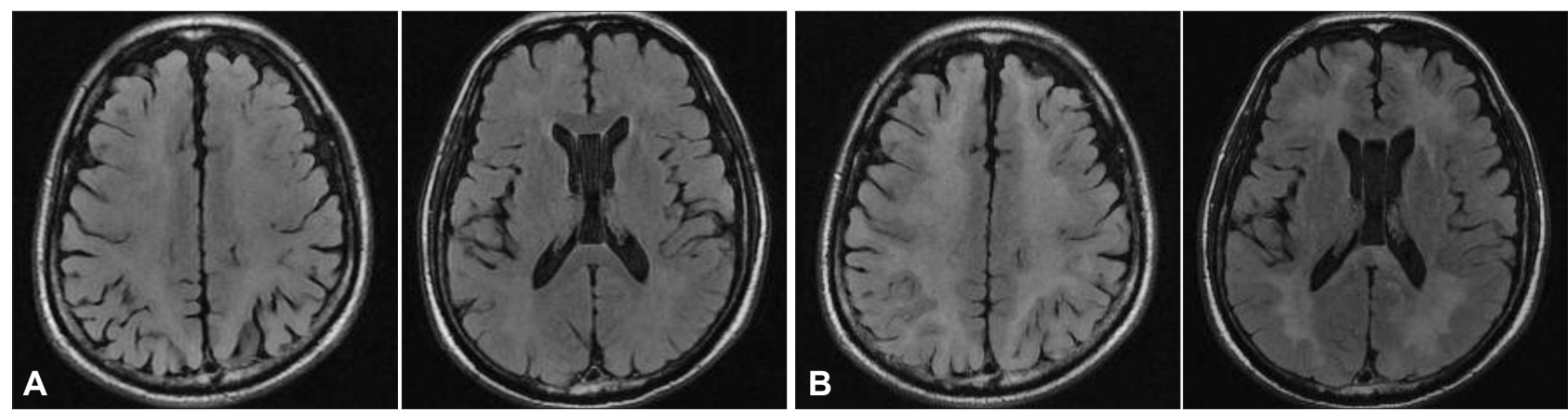

Fig. 2. Case 2; the husband. A: Similar to the magnetic resonance imaging (MRI) findings of case 1, the brain MRI revealed hyperintense lesions in the white matter bilaterally, on T2-weighted and fluid attenuated inversion recovery images. B: Follow-up MRI performed 1 month after admission revealed more extensive hyperintense lesions.

ruled out as the cause of this condition. However, we know of no naturally occurring illness with all of the features exhibited by this couple. We therefore believe that exposure to oriental medicines can result in toxic encephalopathies, although we have not unequivocally established a cause-and-effect relationship. Neurologists and related health-care professionals should consider oriental medicine as a possible cause in unresolved cases of toxic leukoencephalopathies.

\section{Conflicts of Interest}

The authors have no financial conflicts of interest.

\section{REFERENCES}

1. Filley CM, Kleinschmidt-DeMasters BK. Toxic leukoencephalopathy. $N$ Engl J Med 2001;345:425-432.
2. Jung KH, Chu K, Kim YA, Jeon BS. Rapidly progressive toxic leukoencephalomyelopathy with myelodysplastic syndrome: a clinicopathological correlation. J Clin Neurol 2007;3:45-49.

3. Ernst E. Serious psychiatric and neurological adverse effects of herbal medicines -- a systematic review. Acta Psychiatr Scand 2003;108:83-91.

4. Rella J, Marcus S, Wagner BJ. Rapid cyanide detection using the Cyantesmo kit. J Toxicol Clin Toxicol 2004;42:897-900.

5. McKinney AM, Kieffer SA, Paylor RT, SantaCruz KS, Kendi A, Lucato L. Acute toxic leukoencephalopathy: potential for reversibility clinically and on MRI with diffusion-weighted and FLAIR imaging. AJR Am J Roentgenol 2009;193:192-206.

6. Nam TS, Choi SM, Kang KW, Kim JT, Lee SH, Park MS, et al. Acute toxic encephalopathy due to the ingestion of Rhus extract. J Clin Neurosci 2009;16:1377-1378.

7. Kim JS, Chung SW, Chung TI, Park JW, Lee KS, Lee JH. Posterior reversible leukoencephalopathy syndrome: possible relation to licorice. Eur J Radiol Extra 2003;46:83-85. 